\title{
Transhepatic Approach for a Small Paracaval Tumor in Repeat Resection
}

\author{
Takeaki Ishizawa Kiyoshi Hasegawa Mami Ikeda Taku Aoki Keiji Sano \\ Hiroshi Imamura Norihiro Kokudo Masatoshi Makuuchi \\ Department of Surgery, Hepato-Biliary-Pancreatic Surgery Division, Graduate School of Medicine, \\ University of Tokyo, Tokyo, Japan
}

\section{Key Words}

Hepatocellular carcinoma $\cdot$ Liver metastases $\cdot$ Liver resection

\begin{abstract}
To resect a small liver tumor located in the paracaval portion, we adopted transhepatic enucleation, i.e. enucleation of the tumor from the transected plane along the main portal fissure. In contrast to caudate lobectomy, this procedure can save dissection around the liver and vena cava, which would increase operation time and blood loss especially in repeat surgery after removal of Couinaud's segment VII or the right lateral sector. It can also minimize the liver parenchymal volume to be resected. The transhepatic enucleation would be a safe and recommendable surgical technique for a small paracaval tumor in repeat resection and/or in patients with poor liver functional reserve.

Copyright $\odot 2007$ S. Karger AG, Basel
\end{abstract}

\section{Introduction}

Because the paracaval portion of the liver lies deep and is surrounded by the major vessels (the middle and right hepatic veins, the inferior vena cava) and the hepatic hilum $[1,2]$, it is difficult to resect paracaval tumors. Several procedures have been used to resect paracaval tu- mors [3]: combined resection of the overlying hemiliver, sector, or segment $[4,5]$, and caudate lobectomy by high dorsal resection [6] or the anterior transhepatic approach $[7,8]$. However, the combined resection of an overlying segment(s) for small paracaval tumors has the disadvantage of sacrificing a large functional liver mass. Caudate lobectomy is another possible approach, but it is complicated in patients with recurrence after resection that includes Couinaud's segment VII, because of the need to dissect the former raw surface and to fully mobilize the liver.

In this report we describe enucleation of a small paracaval tumor from a plane of transection along the main portal fissure as an effective alternative in repeat surgery.

\section{Patients and Methods}

Patient 1

A 47-year-old male was admitted to our hospital in July 1999 for a third liver resection, this time for two nodules of recurrent hepatocellular carcinoma (HCC) associated with chronic hepatitis $\mathrm{B}$. One nodule measured $1.3 \mathrm{~cm}$ in diameter, and was located in the paracaval portion, immediately posterior to the middle hepatic vein (MHV, fig. 1A), and the other measured $2.5 \mathrm{~cm}$ in diameter and was located in segment II. Right lateral sectoriectomy had been performed to treat the primary HCC in 1997, and limited resection has been performed for a recurrence in segment III in 1998. Before the third operation, the patient's liver function

\section{KARGER}

Fax +4161306 1234 E-Mail karger@karger.ch www.karger.com (c) 2007 S. Karger AG, Basel

0253-4886/07/0246-0409\$23.50/0

Accessible online at:

www.karger.com/dsu
Norihiro Kokudo, MD, PhD

Hepato-Biliary-Pancreatic Surgery Division, Department of Surgery

Graduate School of Medicine, University of Tokyo

7-3-1 Hongo, Bunkyo-ku, Tokyo 113-8655 (Japan)

Tel. +81 35800 8844, Fax +81 35800 8844, E-Mail KOKUDO-2SU@h.u-tokyo.ac.jp 

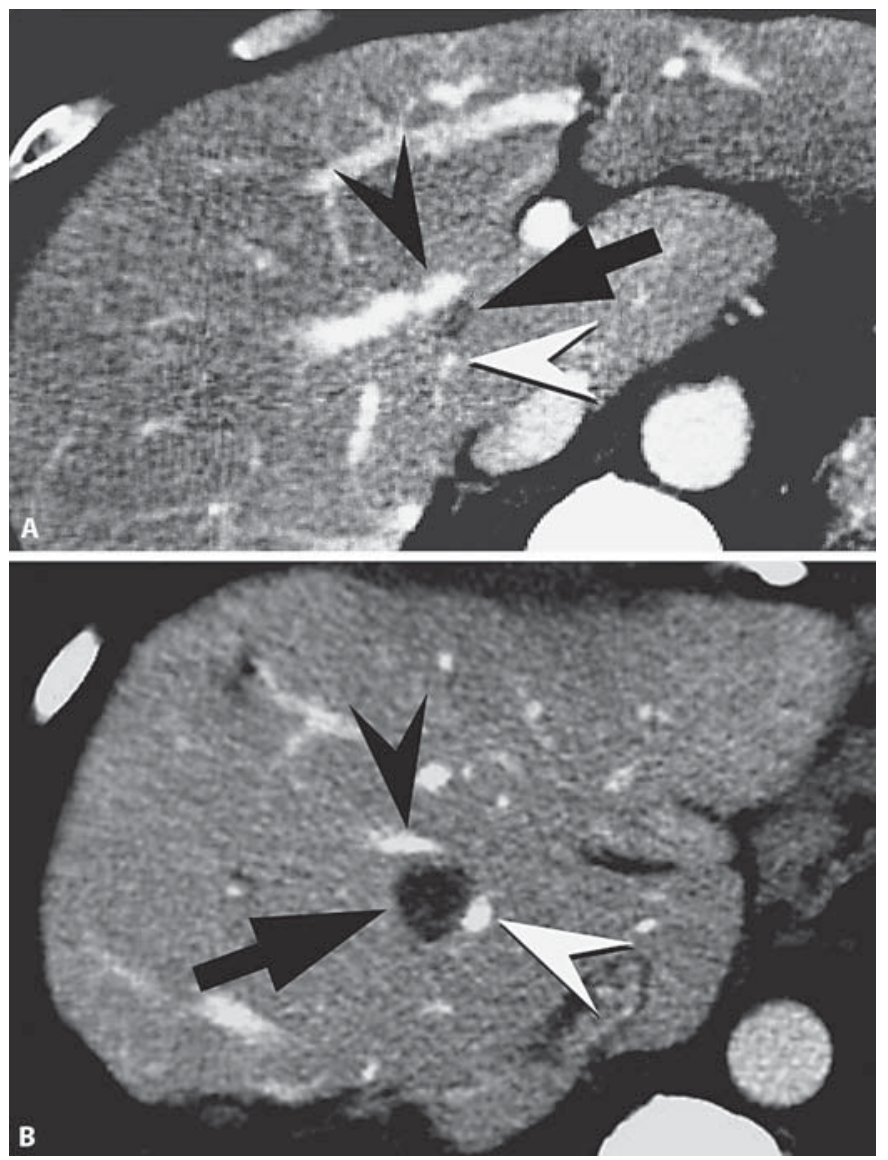

Fig. 1. Computed tomography before surgery in patient $1(\mathbf{A})$ and patient 2 (B). The tumor (arrow) located in the right paracaval portion, between the MHV (black arrowhead) and portal vein running toward the paracaval portion (white arrowhead).

was classified as Child-Pugh class A, and the indocyanine green retention rate at $15 \mathrm{~min}$ (ICGR15) was $17.4 \%$. The volume of the caudate lobe, left lobe, and segment IV accounted for 8, 35, and $12 \%$, respectively, of total liver volume.

After resection of segment II, we decided to enucleate the paracaval tumor via the transhepatic approach to avoid dissection of severe adhesions that had developed after the previous operations and to preserve as much of the liver as possible. First, the liver parenchyma was widely divided, about $12 \mathrm{~cm}$ in length, along the main portal fissure. We preserved the MHV on the right paramedian sector and exposed the left wall of the MHV. At this point, the border of the tumor could be palpated just beneath the raw surface on the right paramedian sector. Then, guided by intraoperative ultrasonography, we enucleated the tumor through the raw surface, maintaining a minimal surgical margin.

The operation time was $320 \mathrm{~min}$. Intraoperative blood loss was $730 \mathrm{ml}$, and transfusion was unnecessary. The postoperative course was uneventful, but a fourth resection, for a recurrence in segment VIII, was performed 14 months later. The patient is currently alive and recurrence-free 54 months after the fourth surgery.



Fig. 2. Intraoperative photography and schema in patient 2 after completion of transhepatic enucleation of the paracaval tumor. A The remnant liver in situ. Arrow shows the dissection line along the main portal fissure. Arrowheads indicate the surgical scar due to the previous surgery. $\mathbf{B}$ The transected planes on the main portal fissure were opened. The MHV (arrow in the schema) was exposed preserving the main tributaries draining the right and left paramedian sectors (arrowheads). The depression on the raw surface of the right side of MHV can be seen, due to enucleation of the tumor in the paracaval portion (asterisk).

\section{Patient 2}

In August 2005, a 62-year-old male was admitted to undergo a second liver resection, this time for a recurrent tumor, $1.7 \mathrm{~cm}$ in diameter, located immediately posterior to the MHV (fig. 1B). In May 2005, he had undergone a right hemicolectomy for cecal carcinoma and limited liver resections for four synchronous metastases in segments II, IV, VI, and VII. The serum albumin and total bilirubin levels and the ICGR 15 value before the second operation were $3.4 \mathrm{~g} / \mathrm{dl}, 0.6 \mathrm{mg} / \mathrm{dl}$, and $10.1 \%$, respectively. The volume of the caudate lobe and the left liver were estimated to be 5 and $30 \%$, respectively, of the total liver volume.

Because the paracaval tumor was small, we decided to perform transhepatic enucleation. The liver parenchyma was widely transected for about $10 \mathrm{~cm}$ along the main portal fissure. The MHV was preserved on the left liver exposing the right wall on the raw 
surface. The right wall and the posterior wall of the MHV in the portion where the tumor was attached to the MHV were carefully dissected from the tumor. After complete dissection of the tumor from the MHV, the tumor was enucleated from the raw surface of the right paramedian sector (fig. 2A, B).

The operation time was $665 \mathrm{~min}$. Blood loss was $510 \mathrm{ml}$, and transfusion was not required. Although a cerebral hemorrhage occurred on postoperative day 4 , the patient was treated conservatively and recovered well. He underwent a third liver resection, for four recurrences in segments II, IV, VI, and VII, 4 months later.

In both patients the liver parenchyma was divided by the clamp-crushing method under intermittent inflow occlusion. Even though a biliary decompression tube was not inserted, no bile leaks occurred in either patient. Surgical margins were negative for cancer, and there have been no local recurrences.

\section{Discussion}

Our experience in these 2 cases showed that a small paracaval tumor can be safely resected by transhepatic enucleation, i.e., enucleation from the transected plane along the main portal fissure. The key to success is making the dissection plane wide enough to approach the deeply situated tumor from the raw surface. The liver parenchyma is dissected from the diaphragmatic surface, exposing the wall of the MHV. The major MHV tributaries draining the opposite side of the liver can be preserved. Intraoperative ultrasonography is indispensable to understanding the interrelationships among the tumor, MHV, hepatic hilum, and the surgical margin.

The major advantage of this procedure is that, in contrast to caudate lobectomy [6-8], mobilization of the right and left liver is unnecessary, thereby saving time and decreasing blood loss. In spite of the deep tumor location and severe adhesions around the liver, the operation time and blood loss in our 2 patients $(320 \mathrm{~min}$ and $730 \mathrm{ml}$, respectively, and $665 \mathrm{~min}$ and $510 \mathrm{ml}$, respectively) were comparable to the operation time and blood loss in our previous series (median values of $360 \mathrm{~min}$ and $635 \mathrm{ml}$, respectively, in the $532 \mathrm{HCC}$ patients, and 408 min and 578 $\mathrm{ml}$, respectively, in the 262 with other liver malignancies) [9]. Furthermore, in patients who have previously undergone liver resection that included segment VII, dissection of adhesions around the right liver may even critically injure the vena cava. Thus, the transhepatic approach, which avoids risky dissection, is especially useful for patients with a recurrent tumor, as in our 2 patients.

Another advantage is that transhepatic enucleation minimizes loss of functional liver parenchyma. The conventional techniques $[4,5]$ remove the overlying seg- ment(s) together with the paracaval portion, but they are invasive and oncologically unnecessary for small paracaval tumors. Our procedure can be applied to patients whose resectable liver volume is greatly restricted because of poor liver function, or because of multiple resections required for several tumors. In patient 1 , the transhepatic enucleation allowed safe resection of both the paracaval HCC and the HCC in segment II. An alternative procedure could have been left hepatectomy, but that would have been too extensive in our first patient in view of the decreased liver function and small tumor size.

Repeat liver resection is acknowledged to be the most effective treatment for recurrence of HCC $[10,11]$ and of colorectal metastasis $[12,13]$. Thus, transhepatic enucleation should provide a survival benefit for patients with HCC or a metastatic tumor in the paracaval portion, not only by permitting resection of the current tumor, but by preserving the functional liver volume for future resections for recurrence. Actually, both patients experienced an intrahepatic recurrence, but successful repeat resection was possible because of the preserved liver function.

Our technique should also be useful as bridging therapy for liver transplantation, although it was not used for that in the 2 cases described. The less invasive surgery allowed by this anterior approach should contribute to maintaining both liver function and tumor-free status during the waiting period.

Since advances in imaging modalities will provide more frequent opportunities to detect recurrent liver tumors at an earlier stage $[14,15]$, transhepatic enucleation should increase the number of candidates for resection of a small paracaval tumor instead of more invasive procedures. However, enucleation of a large paracaval tumor extending to the right and/or left paramedian sector from the transected plane is difficult, and the risk of tumor exposure or rupture is high. Such tumors must be resected with the surrounding liver parenchyma.

In conclusion, transhepatic enucleation is a safe surgical technique and can be recommended for small paracaval tumors in repeat liver resections.

\footnotetext{
References

1 Kumon M: Anatomy of the caudate lobe with special reference to portal vein and bile duct. Acta Hepatol Jpn 1985;26:1193-1199.

$\checkmark 2$ Kogure K, Kuwano H, Fujimaki N, Makuuchi M: Relation among portal segmentation, proper hepatic vein, and external notch of the caudate lobe in the human liver. Ann Surg 2000;231:223-228.
} 
3 Makuuchi M, Kawasaki S, Takayama T, Kumon M: Caudate lobectomy; in Lygidakis NJ, Makuuchi M (eds): Pitfalls and Complications in the Diagnosis and Management of Hepatobiliary and Pancreatic Diseases: Surgical, Medical, and Radiological Aspects. Stuttgart, Thieme, 1993, pp 124-132.

-4 Yamamoto H, Nagino M, Kamiya J, Hayakawa N, Nimura Y: Surgical treatment for colorectal liver metastases involving the paracaval portion of the caudate lobe. Surgery 2005; 137:26-32.

5 Hawkins WG, DeMatteo RP, Cohen MS, Jamagin WR, Fong Y, D'Angelica M, et al: Caudate hepatectomy for cancer: a single institution experience with 150 patients. J Am Coll Surg 2005;200:345-352.

-6 Takayama T, Tanaka T, Higaki T, Katou K, Teshima Y, Makuuchi M: High dorsal resection of the liver. J Am Coll Surg 1994;179: 72-75.
Yamamoto J, Takayama T, Kosuge T, Yoshida J, Shimada K, Yamasaki S, et al: An isolated caudate lobectomy by the transhepatic approach for hepatocellular carcinoma in cirrhotic liver. Surgery 1992;111:699-702.

$\checkmark 8$ Kosuge T, Yamamoto J, Takayama T, Shimada K, Yamasaki S, Makuuchi M, et al: An isolated, complete resection of the caudate lobe, including the paracaval portion, for hepatocellular carcinoma. Arch Surg 1994;129: 280-284.

-9 Imamura H, Seyama Y, Kokudo N, Maema A, Sugawara Y, Sano K, et al: One thousand forty-six hepatectomies without mortality in 8 years. Arch Surg 2003;138:1198-1206.

10 Minagawa M, Makuuchi M, Takayama T, Kokudo N: Selection criteria for repeat hepatectomy in patients with recurrent hepatocellular carcinoma. Ann Surg 2003;238:703710.

11 Poon RTP, Fan ST, Wong J: Risk factors, prevention, and management of postoperative recurrence after resection of hepatocellular carcinoma. Ann Surg 2000;232:10-24.
12 Minagawa M, Makuuchi M, Torzilli G, Takayama T, Kawasaki S, Kosuge T, et al: Extension of the frontiers of surgical indications in the treatment of liver metastases from colorectal cancer: long-term results. Ann Surg 2000;231:487-499.

13 Shaw IM, Rees M, Welsh FKS, Bygrave S, John TG: Repeat hepatic resection for recurrent colorectal liver metastases is associated with favourable long-term survival. Br J Surg 2006;93:457-464.

14 Murakami T, Kim T, Takamura M, Hori M, Takahashi S, Federle MP, et al: Hypervascular hepatocellular carcinoma: detection with double arterial phase multi-detector row helical CT. Radiology 2001;218:763-767.

15 Kim KW, Kim AY, Kim TK, Park SH, Kim HJ, Lee YK, et al: Small $(\leq 2 \mathrm{~cm})$ hepatic lesions in colorectal cancer patients: detection and characterization on mangafodipir trisodium-enhanced MRI. Am J Roentgenol 2004;182:1233-1240 